\title{
Cerebral and Intra-ventricular Abscess Caused by Bisphosphonate-related Osteonecrosis of the Jaw (BRONJ)
}

\author{
Atsushi Kobayashi, ${ }^{1}$ Goro Nagashima, ${ }^{1,2}$ Masayuki Noda, ${ }^{1}$ Akihito Kato, ${ }^{2}$ Hiroyuki Morishima, ${ }^{1}$ Hiroaki Ishii, ${ }^{3}$ \\ Hiroyuki Kunishima, ${ }^{4}$ and Hiroshi Matsumoto ${ }^{5}$
}

\begin{abstract}
Bisphosphonate-related osteonecrosis of the jaw (BRONJ) is one of the major complications caused by prolonged bisphosphonate administration. We treated a case of BRONJ-related cerebral and intraventricular abscess. An 80-year-old woman was referred to our hospital for osteonecrosis of the maxilla. Removal of a decayed tooth followed by several oral antimicrobial administrations was performed, but the inflammation spread gradually into the orbit. Twenty-seven months after the initial treatment, she was referred to our hospital. A computed tomography (CT) scan revealed formation of an intraventricular abscess secondary to destruction of the maxillary and sphenoid sinuses. Reports of BRONJ associated with intracranial infection are rare. With prolonged life expectancy, BRONJ cases will increase with many comorbid diseases. Co-operation among physicians, dentists, and pharmacologists will be needed to treat these conditions appropriately.
\end{abstract}

Keywords: BRONJ, cerebral, intraventricular, abscess

\section{Introduction}

Bisphosphonate-related osteonecrosis of the jaw (BRONJ) was first described in 2003 as a severe complication caused by prolonged intravenous bisphosphonate use. ${ }^{1)}$ It is said that odontogenic infections may become a trigger for BRONJ, but appropriate treatment has not been developed.

In this article, we present a case of BRONJ-related cerebral and intraventricular abscess, and discuss its importance in the era of prolonged life expectancy.

\section{Case Report}

An 80-year-old woman was referred to our hospital from a dental clinic. She had experienced subarachnoid hemorrhages several times, and predonine $5 \mathrm{mg} /$ day and bisphosphonate (Minodronic acid hydrate) $50 \mathrm{mg} / \mathrm{month}$ were prescribed for her rheumatoid arthritis and osteoporosis for more than 3 years, orally. There was no neurological deficit other than mild dementia and her ability of daily living was independent at this time. Following a diagnosis of osteonecrosis of the maxilla, a decayed tooth was removed using

Departments of ${ }^{1}$ Neurosurgery, ${ }^{2}$ Emergency and Disaster Medical Care Center, ${ }^{3}$ Oral and Maxillofacial Surgery, ${ }^{4}$ General Internal Medicine, and ${ }^{5}$ Pharmacy, St. Marianna University Kawasaki Municipal Hospital, Kawasaki, Kanagawa

Received: March 19, 2015; Accepted: June 17, 2015 oral ampicillin. One month after the tooth removal, palate and gingival swelling were recognized, and the antibiotics were changed to cefdinir followed by levofloxacin. Fig. 1 shows the swollen gingiva 5 months later; the antibiotics were discontinued at this point. Cefdinir was re-started for the recurrence of local inflammation 6 months later, and then changed to faropenem. CT findings at this point are presented in Fig. 2a. The inflammation was localized to the maxillary sinus. Nine months after this, a CT revealed inflammation extending into the orbit and soft tissue under the skin of the face (Fig. 2b, c). Only dental treatment without antimicrobials was continued thereafter.

Twenty-seven months after the initial tooth removal, the patient was transferred to our hospital by ambulance. With a diagnosis of septic shock, intensive treatment was started.

A CT scan revealed the destruction of the maxillary and sphenoid bones, and hydrocephalus. A magnetic resonance imaging (MRI) revealed the formation of an intraventricular abscess (Fig. 3).

The patient's white blood cell count was $15,300 / \mathrm{m}^{2}$, C-reactive protein was $13.6 \mathrm{mg} / \mathrm{dl}$, cerebrospinal fluid (CSF) cell count was 19,653/3, and glucose level in her CSF was $0 \mathrm{mg} / \mathrm{dl}$.

Meropenem and vancomycin were started, and intraventricular irrigation and ventricular drainage were performed. Fig. 4 shows the CSF content and endoscopic appearance in the ventricle. Endoscopically, the ventricle was filled with white cotton-like material, and the CSF was turbid with

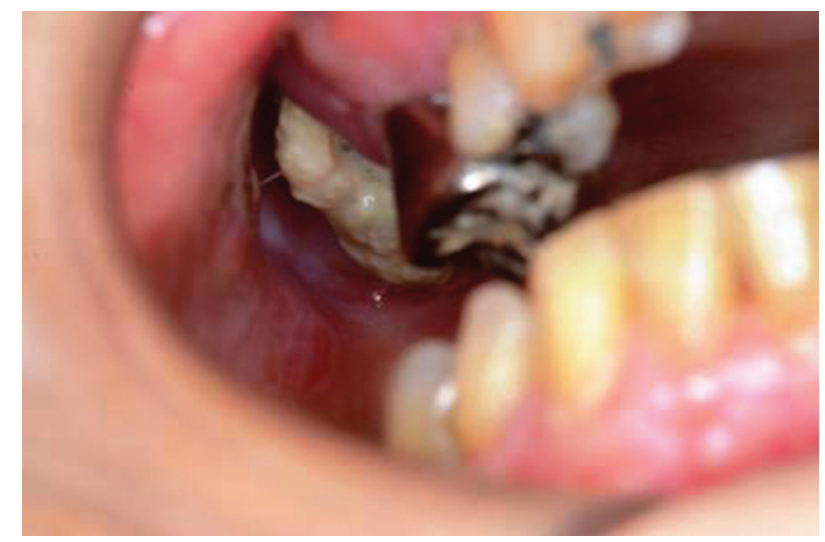

Fig. 1 Photograph of swollen, inflamed gingiva 5 months after the initial tooth removal is shown. Antibiotic treatment was discontinued thereafter. 


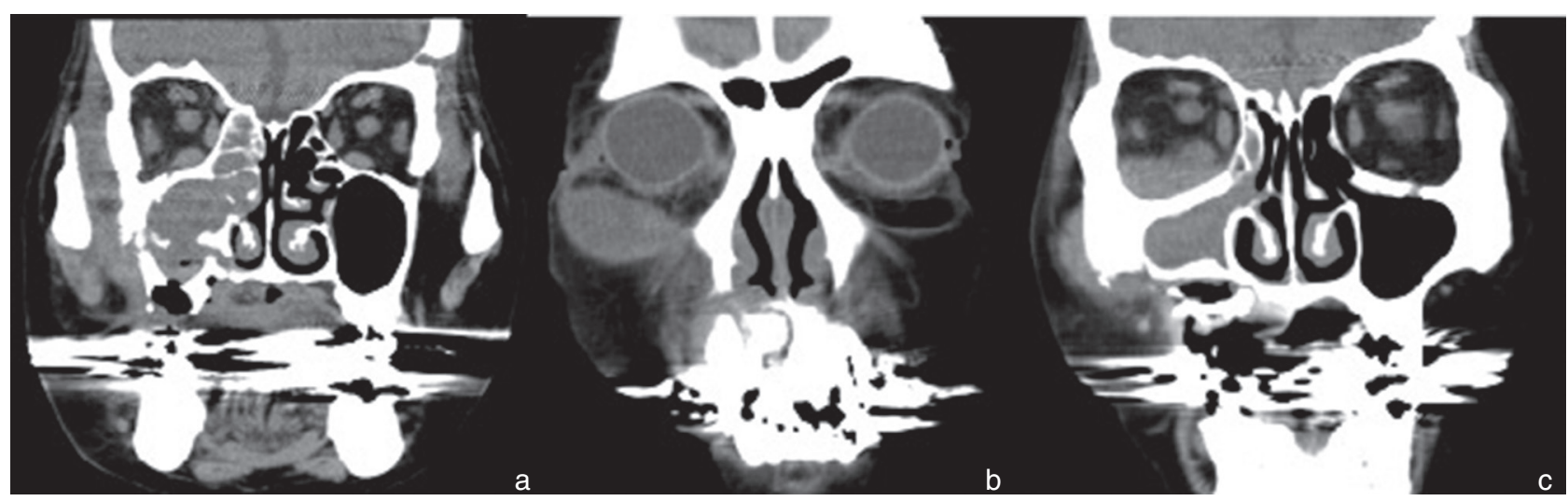

Fig. 2 a: Computed tomography (CT) scan 6 months after treatment shows the inflammation is localized to the maxillary sinus. b, c: CT scan 9 months after the initial treatment shows the inflammation extending into the orbit and soft tissue under the skin of the face.
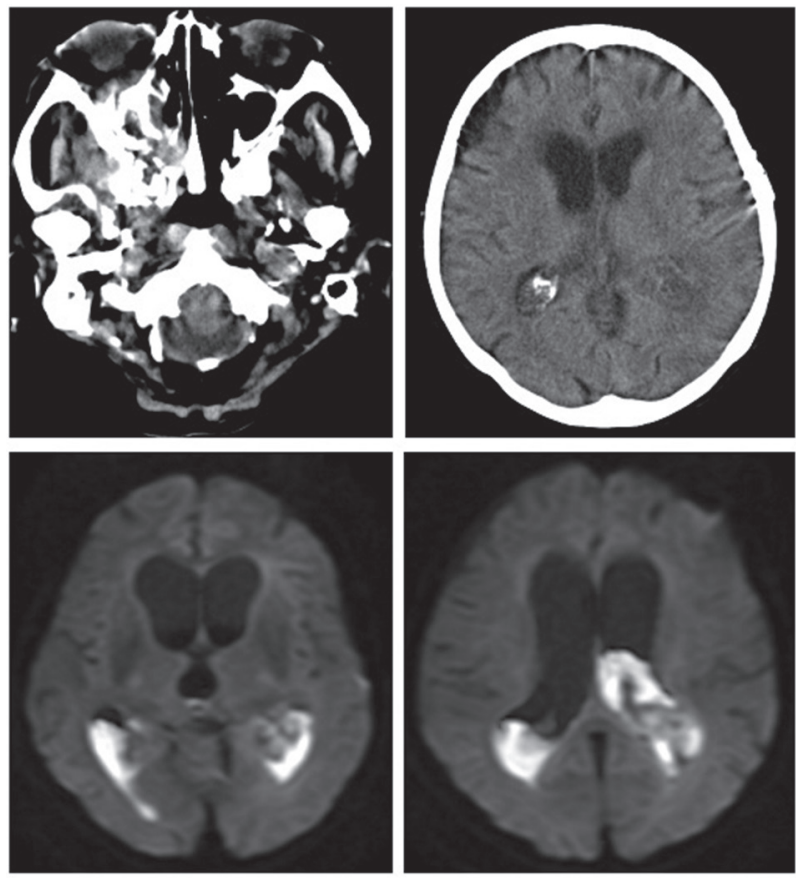

Fig. 3 Twenty-seven months after the initial tooth removal, the patient was transferred to our hospital. A computed tomography scan revealed destruction of the maxillary and sphenoid bones, and hydrocephalus (upper). Magnetic resonance imaging revealed intraventricular abscess formation (lower).

white pus. Bacteroides pyogenesis was recovered from the CSF. After 2 months of antimicrobial administration, a ventriculo-peritoneal shunt was performed (Fig. 5), and she was transferred to a nursing home. Because the patient became bed-ridden along with these treatments, further aggressive treatment against BRONJ was not accepted from her relatives.

\section{Discussion}

BRONJ was first described in 2003. It is thought that odontogenic infections expose patients to considerable risk of BRONJ. Appropriate treatments have not been developed
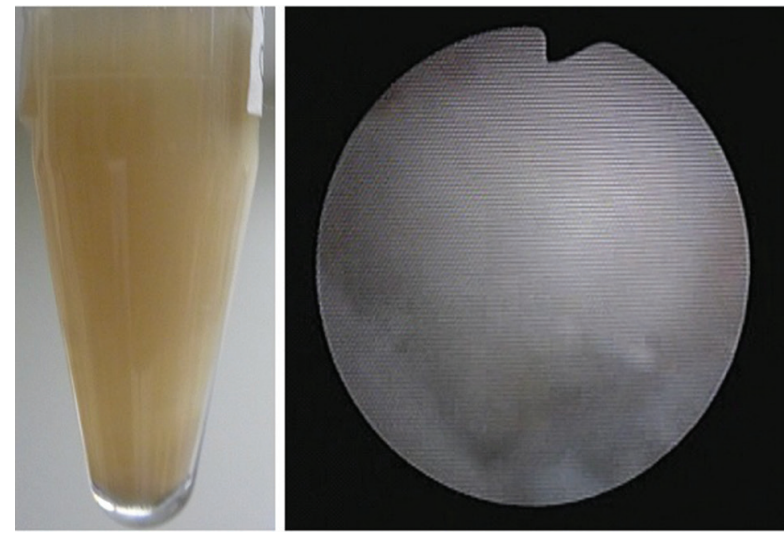

Fig. 4 Photographs during ventricular irrigation are shown. Cerebrospinal fluid was turbid with white ilel, and endoscopically the ventricle was filled with white cotton-like material.
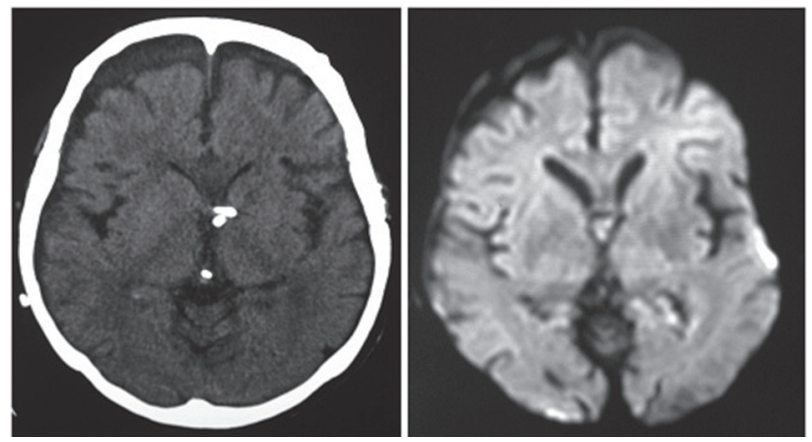

Fig. 5 After 2 months of antimicrobial administration, a ventriculoperitoneal shunt was performed, and the patient was transferred to a nursing home. Ventricular tubes were inserted bilaterally into the lateral ventricles (left). An MRI diffusion image confirms the absence of infection (right). MRI: magnetic resonance imaging.

so far, and the role of infection in the etiology of BRONJ is poorly understood. ${ }^{1)}$

Hinson et al. reported that Actinomyces plays a key role in the formation of osteonecrosis, and more than half of the recovered organisms are Streptococci. ${ }^{2)}$ They recommended 
obtaining hematoxylin and eosin (H\&E), periodic acid Schiff (PAS), and Gram stains, along with the requirement of a tissue reaction in the immediate vicinity, to differentiate colonization versus infection, for biopsy tissue obtained from BRONJ patients. Updated 2009 guidelines suggest that combined surgical intervention (removing necrotic bone) along with antimicrobial rinses and empiric systemic antibiotic coverage (treating infected, viable bone) will improve healing with Stages I and II of the disease. Patients presenting with Stage III likely will benefit from surgical debridement in combination with some form of antibiotic therapy. ${ }^{3)}$ The "CROMa" project describes patients' care pathways, but appropriate treatment against infection has not been described. ${ }^{4)}$ Ikeda et al. described the effect of sitafloxacin for treatment of BRONJ, analyzing 20 cases of BRONJ. ${ }^{5)}$ They stated that every patient had at least one bacterial species that showed resistance to common antibiotics. In their study, Streptococci were recovered in 14 cases, Prevotella species in 6 cases, Parvimonas species in 5 cases. Steroid effects on BRONJ also have been reported. Manzon et al. reported a case of rheumatoid arthritis treated with bisphosphonate and corticosteroid, which developed into a temporal abscess caused by Actinomyces. Meropenem and metronidazole were selected as the antimicrobials for the treatment. ${ }^{6)}$ Bullock and Fleishman reported the importance of BRONJ with extended infection to the central nervous system. ${ }^{7)}$ Jokipii and Jokipii reported that anaerobic bacteria were isolated from $31 \%$ of ears with chronic otitis media and from $28 \%$ (or $48 \%$ if microaerophilic Streptococci were included) of chronic maxillary sinusitis cases. Anaerobic central nervous system infections are typically caused by Bacteroides species and microaerophilic Streptococci ${ }^{8)}$ In this case, bisphosphonates and steroids were used for more than 3 years, and were thought to be major risk factors of BRONJ. We must take these organisms into account to treat BRONJ-related central nervous system infections.

Reports about BRONJ associated with intracranial infection are rare. With prolonged life expectancy, BRONJ cases will increase accompanied by many severe comorbid diseases. Guidelines for surgical management and antimicrobial selection must be developed considering the extension of the disease into the central nervous system. Physicians, dentists, and pharmacologists must learn more about BRONJ, and co-operation against these diseases will be needed.

\section{Conflicts of Interest Disclosure}

The authors have no personal, financial, or institutional interest in any of the drugs, materials, or devices in the article. All authors who are members of the Japan Neurosurgical Society (JNS) have registered online Self-reporting Conflict Disclosure Statement Forms through the website for JNS members.

\section{References}

1) Marx RE: Pamidronate (Aredia) and zoledronate (Zometa) induced avascular necrosis of the jaws: a growing epidemic. J Oral Maxillofac Surg 61: 1115-1117, 2003

2) Hinson AM, Smith CW, Siegel ER, Stack Jr BC: Is bisphosphonaterelated osteonecrosis of the jaw an infection? A histological and microbiological ten-year summary. Int J Dent 2014: 452737, 2014

3) Hoefert S, Eufinger H: Relevance of a prolonged preoperative antibiotic regime in the treatment of bisphosphonate-related osteonecrosis of the jaw. J Oral Maxillofac Surg 69: 362-380, 2011

4) Capocci M, Romeo U, Cocco F, Bignozzi I, Annibali S, Ottolenghi L: The "CROMa" project: a care pathway for clinical management of patients with bisphosphonate exposure. Int J Dent 2014: 719478, 2014

5) Ikeda T, Kuraguchi J, Kogashiwa Y, Yokoi H, Satomi T, Kohno N: Successful treatment of bisphosphonate-related osteonecrosis of the jaw (BRONJ) patients with sitafloxacin: new strategies for the treatment of BRONJ. Bone 73: 217-222, 2015

6) Manzon L, Ettorre E, Viscogliosi G, Ippoliti S, Filiaci F, Ungari C, Fratto G, Agrillo A: Bisphosphonate therapy and osteonecrosis of the jaw complicated with a temporal abscess in an elderly woman with rheumatoid arthritis: a case report. Clin Interv Aging 9: 1409-1413, 2014

7) Bullock JD, Fleishman JA: The spread of odontogenic infections to the orbit: diagnosis and management. J Oral Maxillofac Surg 43: 749-755, 1985

8) Jokipii AM, Jokipii L: Metronidazole, tinidazole, ornidazole and anaerobic infections of the middle ear, maxillary sinus and central nervous system. Scand J Infect Dis Suppl 26: 123-129, 1981

Corresponding author:

Goro Nagashima, MD, Department of Neurosurgery, St. Marianna University Kawasaki Municipal Hospital, 1-30-37 Shukugawara, Tama-ku, Kawasaki-city, Kanagawa 214-8525, Japan.

$\triangle$ goro-n@marianna-u.ac.jp 